\title{
Characterizing the deterministic nature of individual power dropouts in semiconductor lasers subject to delayed feedback
}

\author{
K. Hicke, X. Porte, and I. Fischer \\ Instituto de Física Interdisciplinar y Sistemas Complejos, IFISC (UIB-CSIC), \\ Campus Universitat de les Illes Balears, E-07122 Palma de Mallorca, Spain
}

(Received 1 August 2013; revised manuscript received 7 October 2013; published 7 November 2013)

\begin{abstract}
We implement a method to identify the deterministic nature of specific events in the dynamics of a semiconductor laser subject to time-delayed optical feedback. Specifically, we study the power dropouts in the low-frequency fluctuations regime on an individual event basis and identify whether the underlying dominant mechanism is deterministic. Our approach is based on sychronization with a twin system in a symmetric relay configuration. We investigate the dependence of the fraction of deterministically driven (i.e., synchronized) dropouts on the laser's pump current as a key parameter. Our experimental results are corroborated by numerical modeling based on rate equations. Our numerical findings also provide insights into the influence of spontaneous emission noise.
\end{abstract}

DOI: 10.1103/PhysRevE.88.052904

PACS number(s): 05.45.Xt, 42.55.Px, 42.65.Sf

\section{INTRODUCTION}

Instabilities in the emission of semiconductor lasers have been studied for a long time. Semiconductor lasers with delayed feedback exhibit a variety of complex dynamical behaviors and these systems serve as excellent test beds for the study of delay-dynamics, chaos synchronization, and applications based thereon (see, e.g., [1]). The dynamics of semiconductor lasers subject to time-delayed feedback is affected by an intricate interplay between deterministic mechanisms and intrinsic noise, the latter resulting from spontaneous emission and carrier fluctuations. Operating such a laser system with moderate to strong feedback in the vicinity of its lasing threshold leads to a characteristic and widely studied dynamical regime called low-frequency fluctuations (LFF). It is characterized by irregular sudden power dropouts and a more gradual recovery of the average emitted power. The dropout can be considered the end of a dynamical cycle. An exemplary experimental time series of an LFF structure is shown in Fig. 1. Each cycle is a power-buildup process while the trajectory itinerates through multiple external cavity modes (ECMs) toward the high-gain region, followed by a sudden drop in intensity and jump back towards the solitary laser frequency. [2,3]. There has been a long and controversial discussion about the origin of LFF dynamics and whether the overall structure and the power dropouts are dominated by deterministic mechanisms or by stochastic processes [2,4-12].

Numerical studies have shown that the qualitative features of the LFF regime in semiconductor lasers can be obtained without intrinsic noise. This underlines that the power dropouts can be evoked by the underlying delay-induced chaotic dynamics only and do not necessarily require noise. Nevertheless, noise could indeed affect the dynamics and induce dropout events, thereby also shortening the LFF cycle compared to a noiseless situation. So far, all investigations to identify whether deterministic mechanisms or stochastic processes dominate the dropout behavior have been based on statistical measures. In this paper, we introduce an individual event-based approach allowing one to identify on an individual basis which power dropouts in the LFF regime are deterministically induced by the feedback signal and not by laser-intrinsic noise.

Our method is based on the synchronization with a twin laser system. It has been shown, that the dynamics of a single chaotic system with delayed feedback is equal to the dynamics of two identical coupled twin systems that are synchronized, i.e., the chaotic attractor in the synchronization manifold of the coupled system is equal to the attractor of the single system with feedback. For our approach to work, the subsystems have to be able to synchronize identically which depends on their coupling and feedback topology (see, e.g., [13]) and their dynamical regime (see, e.g., [14]). For this reason we operate in the moderate feedback regime and use an as symmetric setup as possible. We employ a relay configuration to couple both lasers $[15,16]$. It has been shown that the implementation of a relay, which provides feedback and coupling for both lasers, allows for identical synchronization of the output intensities in such systems. In case both arms of the configuration have the same optical length, zero-lag synchronization can be achieved [13,15-19]. We now employ synchronization as a test to determine whether a power dropout in one of the lasers has been the result of the deterministic chaotic dynamics or of the intrinsic noise in the laser. We argue that if the lasers' outputs exhibit synchronous power dropouts, the events were induced by a deterministic mechanism. Since each laser has its own noise characteristics, it is highly unlikely that both lasers experience a dropout at the same time. In case both lasers exhibit dropouts but at different times, multiple reasons can be postulated. An often occurring situation is that noise induces a dropout event in one laser and after one coupling delay the other laser exhibits a dropout as well; the lasers resynchronize after that.

Our approach can in general be applied to investigate specific outstanding events in the dynamics of other noisy chaotic systems as well. As a necessary condition, these have to be synchronizable such that the attractor of the synchronized (coupled) system is identical to that of a single system. Further discussion of the applicability of our method can be found in Sec. IV. 


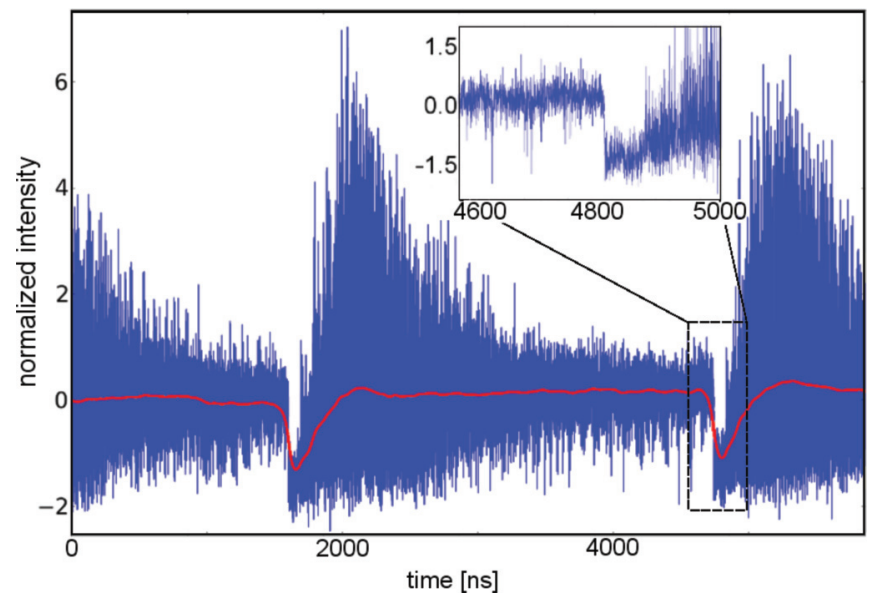

FIG. 1. (Color online) Exemplary experimental time trace of the output intensity fluctuations associated with the LFF regime. The intensity has been shifted by its mean and normalized by its standard deviation. The light (red) curve depicts the time series after applying a low-pass filter. The power buildup and power dropout can be clearly seen at the beginning and the end of the LFF cycle, respectively. The inset shows a magnification of one of the shown dropout events.

We study experimentally and numerically the dependence of the fraction of deterministically driven dropouts $\zeta$ on two parameters that are key in this context: the noise strength and the pump current. With the former we analyze the impact of stochastic processes on the laser dynamics and with the latter we examine how changes in the dynamical regime make the chaotic dynamics susceptible to the effect of a given noise strength.

This paper is organized as follows: Sec. II contains the description of the experiment and the analysis of its results, as well as a discussion about limitations that apply to the described method. In Sec. III we introduce the model we use for our numerical studies and present the corresponding results. In Sec. IV we discuss the applicability to other systems as well as statistical measures to find indications of determinism. Finally, we summarize our results and make final conclusions.

\section{EXPERIMENTS}

Our experimental setup is depicted in Fig. 2. The optical fiber-based coupling configuration consists of two single-mode quantum-well (QW) edge-emitting discrete mode semiconductor lasers (Eblana Photonics), operating at a nominal wavelength of $\lambda \approx 1540 \mathrm{~nm}$ with a side-mode suppression ratio of $>40 \mathrm{~dB}$. The lasers have been hand selected for similar properties.

The lasers are coupled symmetrically via a relay fiber loop. This loop consists of a 50/50-optical coupler and an optical circulator and corresponds to a semitransparent mirror, accounting for symmetric feedback and coupling, respectively, with equal coupling delays and equal coupling and feedback strengths. The two legs of the setup have been equalized in optical path length with an accuracy of the resulting feedback delays of $\left|\tau_{f b, 1}-\tau_{f b, 2}\right| \approx 25 \mathrm{ps}$. We use polarization maintaining (PM) optical fiber; therefore we can exclude effects related to polarization alignment as the origin of desynchronization. The laser temperatures and

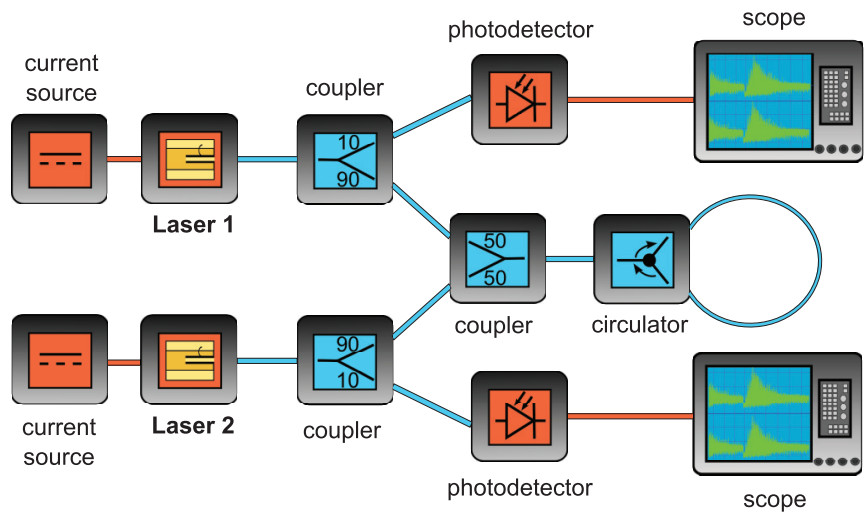

FIG. 2. (Color online) Schematic experimental setup. The lasers are symmetrically coupled via a feedback and coupling fiber loop consisting of a 50/50 optical coupler and a circulator. This loop serves as passive relay. The numbers in the symbols for the couplers correspond to their respective coupling ratios (in percent). The dark (orange) lines depict electrical connections, the light (blue) ones represent polarization-maintaining (PM) optical fiber.

pump currents are controlled by a Thorlabs PRO8000 laser controller with accuracies $\Delta T= \pm 0.01{ }^{\circ} \mathrm{C}$ and $\Delta I= \pm 0.01$ $\mathrm{mA}$, respectively. We measure the laser outputs by using Miteq Dr-125G-A photodetectors with $13 \mathrm{GHz}$ bandwidth, the outputs of which are recorded by a LeCroy WaveMaster $816 \mathrm{Zi}$ oscilloscope with an analog bandwidth of $16 \mathrm{GHz}$ and a sampling rate of $40 \mathrm{GS} / \mathrm{s}$. This corresponds to a temporal resolution of $25 \mathrm{ps}$.

By autocorrelation analysis the two feedback delays in our setup were estimated to be $\tau_{f b, 1}=89.75 \pm 0.025$ and $\tau_{f b, 2}=89.73 \pm 0.025$ ns. Thus, with the coupling delay $\tau_{c}=\frac{1}{2}\left(\tau_{f b, 1}+\tau_{f b, 2}\right)$, all delay times can be considered equal within the uncertainty bounds.

We measured the feedback attenuation along both optical feedback paths as $\approx-11 \mathrm{~dB}$ being equal for both arms within a margin of $1 \%$. Because of this symmetry, the coupling has the same attenuation and thus the same strength. Considering the geometry and coupling of the laser cavities we estimate the total feedback rates (and thus the coupling rates) per laser as $\kappa_{1}=\kappa_{2}=\kappa_{c}=34 \mathrm{~ns}^{-1}$.

Both lasers are being operated in the low-frequency fluctuations regime. This means that the pump current is relatively close to the solitary threshold. The feedback strength corresponds to a few percent of the output power coupled back into the cavity.

The lasing threshold pump currents are $I_{\text {thr1 }}=10.89$ and $I_{\mathrm{thr} 2}=10.92 \mathrm{~mA}$, respectively. We define the normalized pump currents as $p_{1,2}=I_{1,2} / I_{\mathrm{thr} 1,2}$. For our experiments $p_{1}$ and $p_{2}$ are varied simultaneously from $p_{1,2}=1.01$ to $p_{1,2}=1.12$ in steps corresponding to $0.15 \mathrm{~mA}$. For each step, six output intensity time series of both lasers are acquired, each with a length of $100 \mu \mathrm{s}$. Although experimentally challenging due to the optical phase sensitivity of our experimental setup, we identify and only record time series with appropriate phase conditions. The classification as synchronous or nonsynchronous dropouts is done manually for every single measured power dropout because this proved to be the most reliable method. If the dropouts of both lasers occur within a 


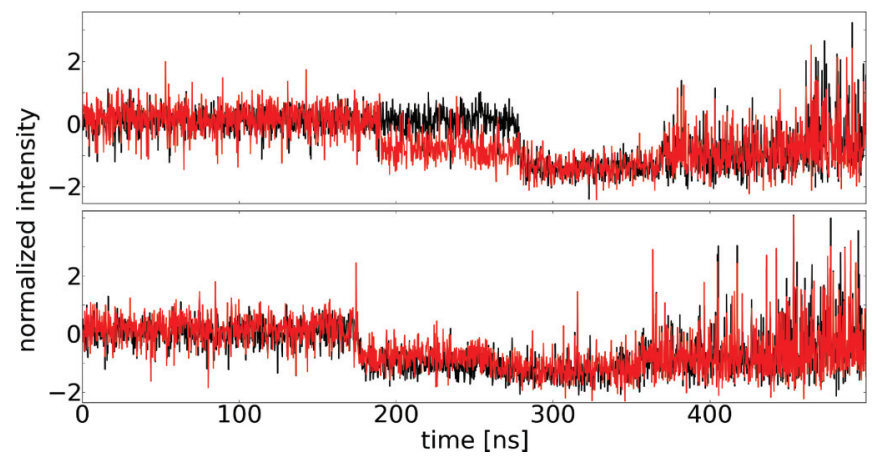

FIG. 3. (Color online) Experimental intensity time traces of both lasers. In the upper panel an unsynchronized dropout is shown. The desynchronization event has a duration corresponding to the delay $\tau_{c} \approx \tau_{f b}$. The lower panel shows a synchronized dropout in both lasers.

2 ns window they are considered as synchronous. Figure 3 shows exemplary time traces of an unsynchronized and a synchronized pair of dropouts, respectively.

To analyze our results we define the total number of detected pairs of dropouts for one set of parameters as $N_{\text {total }}$ and its subset of synchronized pairs of dropouts as $N_{\text {synced }}$. The main measure we use is the synchronized dropout fraction $\zeta$ which is defined as $\zeta=N_{\text {synced }} / N_{\text {total }}$. Our experimental results for $\zeta$ as a function of the normalized applied pump currents $p_{1}$ and $p_{2}$ are depicted in Fig. 4. They exhibit a high fraction ( $\left.\gtrsim 83 \%\right)$ of synchronous dropouts close to the solitary lasing threshold and a decrease with increasing bias current.

This first result is surprising: the fraction $\zeta$ has its maximum just above the lasing threshold. One might have expected a stronger effect of the intrinsic noise close to threshold. This is due to the relatively large contribution of spontaneous emission to the output power close to the lasing threshold. What we observe is contrary to that expectation. We conjecture that the

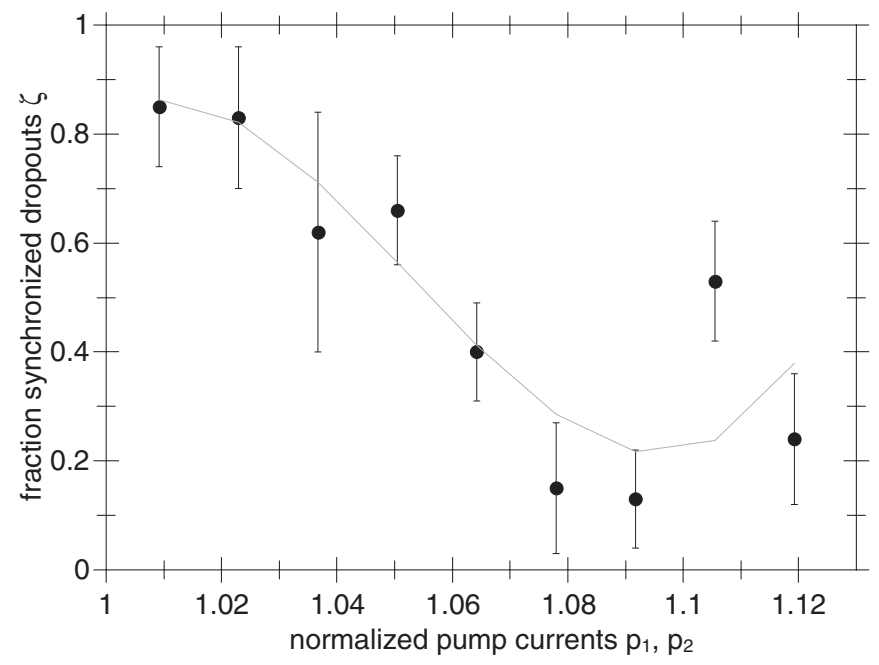

FIG. 4. Experimental results for the fraction of synchronized power dropouts $\zeta$ versus the normalized pump currents $p_{1}$ and $p_{2}$ averaged among the corresponding measured time series (black circles). The error bars show the corresponding standard deviation. The gray curve results from a 3rd order polynomial fit and is only meant as guide to the eyes. noise is not sufficiently strong to induce more power dropouts with the pump current close to threshold than at higher pump currents. This hypothesis is verified via our numerical studies presented in Sec. III.

With the ratio of synchronized dropouts $\zeta$ being this large, we can conclude that close to threshold a significant majority of LFF power dropouts is induced by underlying deterministic mechanisms rather than by noise. Moreover, we can identify which events are deterministic. Since the noise due to spontaneous emission is independent for each laser and correlated noise even for a single round trip is much smaller due to the feedback attenuation and coupling attenuation, we can disregard stochastic effects as the principal drive of power dropouts in the low pump current regime. An analysis of the inter-dropout-interval (IDI) probability distributions with a 2 ns resolution reveals that the chance of coincidental dropouts in both lasers within a 2 ns window is negligible $\left(\ll 10^{-4}\right)$. Once we classify a pair of dropout events as synchronized we can, therefore, name determinism as the dominant mechanism with high confidence.

Considering the lower synchronized dropout fraction for higher pump currents we have to be aware of certain limitations our method has. On the individual event basis we can only classify synchronized dropouts as deterministically driven; we can not determine the main driver of the unsynchronized ones. Attractor bubbling, mismatches in feedback strength and/or delay, mismatches in laser parameters, and detectionrelated misclassifications reduce the fraction of synchronized dropouts without being related to the delayed feedback attractor. Bubbling [20-22] is a desynchronization phenomenon that occurs in coupled systems. In contrast to on-off intermittency [22-25] the synchronization is overall stable; nevertheless the system can exhibit intermittent desynchronization events. Bubbling events are induced by noise and/or parameter mismatch and can be attributed to transversely unstable periodic orbits embedded in the stable synchronization manifold. The local instability forces the system's trajectory to temporarily leave the synchronization manifold in a transverse direction until resynchronization. Bubbling has been investigated theoretically, numerically, and experimentally in lasers [19,22,26]. An increase in the occurrence of bubbling-induced desynchronization with increasing pump current was found, which would, in part, explain the strong decline of the synchronized dropout fraction for increasing pump current. Moreover, a strong connection between the occurrence of bubbling events and that of power dropouts in the LFF regime was discovered. If the lasers are identical and the configuration is symmetric, bubbling occurs only due to transversely unstable saddle nodes embedded in the attractor, not because of the drop in power. The saddles, called antimodes, nevertheless also play an important role for the power dropouts. With slight asymmetries in the experimental conditions and parameters, some of the laser's modes also become transversely unstable and bubbling can occur at different parts of the attractor as well (see, e.g., [13]). In these cases the phase space trajectories diverge and the lasers might exhibit their dropouts at different times due to bubbling.

Pairs of dropouts that are unsynchronized due to bubbling will be (mis-)classified by our approach as not being deterministically driven. Since bubbling-induced desynchronization 
induces dynamics transverse to the synchronization manifold we can make no statement about the principal mechanism underlying these dropouts. Furthermore, certain asymmetries or mismatches in the experimental setup, which in experiments are unavoidable, will lead to more unsynchronized dropouts and therefore to additional misclassifications, since they also can result in dynamics partly outside the synchronization manifold and thus outside the single delay system's dynamics manifold. During the preparation of the experiment, all asymmetries were minimized as much as possible. Although these mismatches still might have an effect, the dynamics within the synchronization manifold is a good approximation for the dynamics of the single delayed feedback system.

There are two further possible sources for misclassification. One is detection noise which contributes to the synchronization error and might blur the dropout event. The second source relates to instances of LFF dynamics where the precise time of occurrence is hard to determine. These uncertainties in the determination of the exact dropout time can arise because the shape of a dropout is not as clear. This happens, in particular, towards higher pump currents, e.g., during the transition from the LFF regime to the fully developed coherence collapse regime where the characteristic shape of the dropouts gets lost.

Considering these misclassification sources the resulting fractions of synchronized dropouts represent a lower limit to the actual values. We conclude that one can not make clear statements about the dominant mechanism underlying the dropouts in the regime where our results show only small to intermediate values for $\zeta$. Since $\zeta$ is highest closest to the solitary threshold, we can draw the strongest conclusions there.

\section{NUMERICAL MODELING}

To model the coupled laser system, we use a rate equation model based on the Lang-Kobayashi equations for a laser subject to time-delayed optical feedback [27]. It is considered adequate for single mode operation of the lasers operating in a moderate feedback regime. The set of rate equations reads

$$
\begin{aligned}
\dot{\mathcal{E}}_{j}(t)= & \frac{1}{2}\left(1+i \alpha_{j}\right)\left[g_{j}\left(n_{j}-n_{T_{j}}\right)-\gamma_{j}\right] \mathcal{E}_{j}(t) \\
& +\kappa_{j} \mathcal{E}_{j}\left(t-\tau_{f b, j}\right)+\kappa_{c} \mathcal{E}_{3-j}\left(t-\tau_{c}\right)+F_{\mathcal{E}_{j}}, \\
n_{j}(t)= & \frac{I_{j}}{e}-\gamma_{e} n_{j}(t)-g_{j}\left(n_{j}-n_{T_{j}}\right)\left|\mathcal{E}_{j}(t)\right|^{2},
\end{aligned}
$$

with the variables $\mathcal{E}_{j}$ and $n_{j}$ representing the slowly varying complex electric field amplitude and the carrier number of the $j$ th laser $(j=1,2)$, respectively. Here, $\alpha$ is the linewidth enhancement factor, $g$ is the differential gain, $n_{T}$ denotes the carrier number at transparency, $\gamma$ is the photon decay rate, $\kappa_{j}$ stands for the feedback rate, $\tau_{f b}$ is the feedback delay, and $\kappa_{c}$ and $\tau_{c}$ are the rate and time delay of the coupling, respectively. $I$ describes the laser pump current, $e$ denotes the elementary charge, and $\gamma_{e}$ is the carrier decay rate.

The noise is modeled as field noise originating from spontaneous emission only; we neglect carrier noise in our approach. The Langevin noise term $F_{\mathcal{E}}$ is modeled as Gaussian white noise with correlation $\left\langle F_{\mathcal{E}}(t) \overline{F_{\mathcal{E}}\left(t^{\prime}\right)}\right\rangle=2 \beta_{j} \gamma_{e} n_{j} \delta\left(t-t^{\prime}\right)$ and zero mean $\left\langle F_{\mathcal{E}}(t)\right\rangle=0$, where $\beta$ is the spontaneous emission factor which describes the fraction of the spontaneously emitted photons that contribute to the lasing mode.

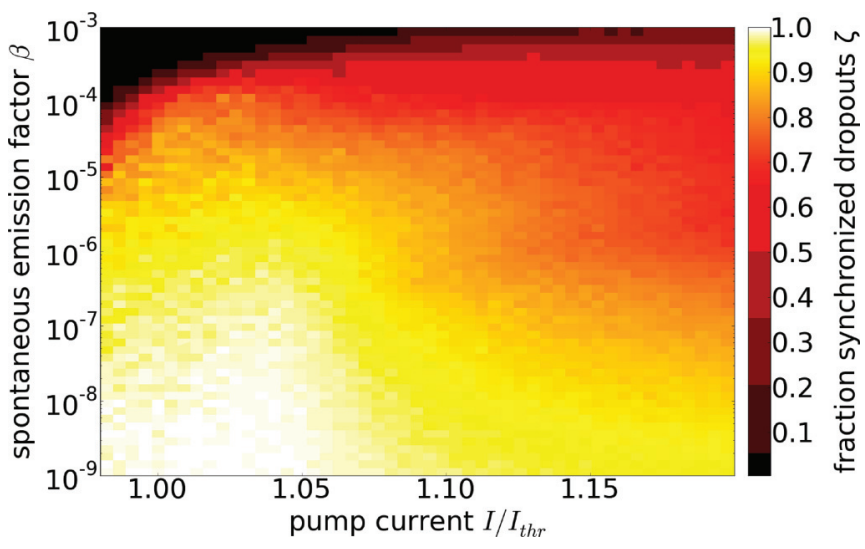

FIG. 5. (Color online) Numerical results for the fraction of synchronized power dropouts $\zeta$ versus the laser's pump current and the spontaneous emission factor. For these results Eqs. (1) and (2) were integrated with symmetric parameter values $\alpha_{j}=3$, $g_{j}=10^{-5} \mathrm{~ns}^{-1}, n_{T_{j}}=2 \times 10^{8}, \gamma_{j}=200 \mathrm{~ns}^{-1}, \kappa_{j}=20 \mathrm{~ns}^{-1}, \tau_{f b, j}=$ $10 \mathrm{~ns}, \kappa_{c}=20 \mathrm{~ns}^{-1}$, and $\tau_{c}=10 \mathrm{~ns}$; all for $j=1,2$.

The equations are numerically solved using Milshtein's method [28,29]. The two coupled lasers are simulated as being identical in every parameter and the coupling scheme is set to be completely symmetrical, neglecting any possible feedback delay or -strength mismatch.

The parameter values of the simulation are given in the caption of Fig. 5. For each set of parameters 20 time series of $10 \mu$ s are computed.

Classification of synchronized and nonsynchronized dropouts is performed automatically for the modeled time series. The dropouts were identified by the following algorithm: The original time series are window averaged with a window size corresponding to the delay time $\tau=10 \mathrm{~ns}$ and a step size of 5 ps. When the intensity drops below the corresponding windowed intensity average for at least $1.7 \mathrm{~ns}$ out of the consecutive 2 ns interval ( 400 points), the occurrence of a power dropout is identified. If a dropout is detected in the time series of both lasers within a $1 \mathrm{~ns}$ window, the drops are considered synchronous. Although this automatized classification may be not as reliable as a manual one, it is sufficiently accurate. Due to the extent of the studied parameter dependencies, a manual classification can not be implemented.

Our numerical results for the synchronized dropout fraction $\zeta$ versus the normalized pump currents and versus the noise strength $\beta$ is depicted in Fig. 5. A spontaneous emission factor of $\beta \approx 10^{-6}$ to $10^{-5}$ is considered realistic for the type of laser used in our experiment.

The results show relatively large fractions of synchronized dropouts for most of the scanned parameter ranges. This can be understood considering the full symmetry. Therefore, mismatch-induced desynchronization is not present. Employing a set of slight mismatches (feedback delays and -strengths, normalized pump currents, and $\alpha$ factors) in our simulations indeed results in significantly lower values for $\zeta$ towards higher pump currents, thus better reflecting the qualitative trend of the experimental results. For small mismatches of the order of $1 \%$ of the aforementioned parameters, we observe a decrease of $\zeta$ to $\zeta \approx 0.4$ for $\beta=10^{-6}$ and $I / I_{\mathrm{thr}}=1.2$ with all other parameters as listed in the caption of Fig. 5. Furthermore, 
classification errors due to experimental limitations can be excluded as well. The numerical findings exhibit maxima of the investigated fractions close to the lasing threshold for small to moderate (realistic) noise strengths and a decrease with increasing pump current. The maximum values for a given noise factor shows a decrease from 1 for $\beta \approx 10^{-9}$ to $\approx 0.1$ for $\beta \approx 10^{-3}$. For noise strengths below $10^{-5}$ the synchronized dropout fraction maximum is close to threshold and decreases with increasing current. For spontaneous emission noise factors above that value the maximum shifts to a larger pump current with the minimum now at or below threshold.

The simulation results corroborate our experimental findings. There is a qualitative agreement with the decrease of the synchronized event fraction with pump current and its maximum closely above threshold. From the numerics we can see the effect of noise close to threshold: with a spontaneous emission factor $\beta \gtrsim 10^{-4}$ which is one to two orders of magnitude stronger than assumed for the experiment, the maximum of the discussed fraction is no longer close to threshold, but at higher pump currents. Stronger noise has a more pronounced effect and can be the dominant driving force close to threshold. With such strong noise the originally expected behavior can be found.

The result that close to threshold the influence of noise on the occurrence of dropouts is minimal can be considered specific to the type of laser we use in the experiment-single mode QW edge emitters. As the spontaneous emission factor $\beta$ can be significantly higher for other laser types, e.g., photonic crystal lasers, the examined fraction can have a minimum close to the lasing threshold with the effect of noise diminishing and thus increasing $\zeta$ with increasing pump current.

\section{DISCUSSION AND CONCLUSIONS}

We have presented an approach to identify deterministically induced power-dropout events in semiconductor lasers subject to time-delayed feedback. The method is based on synchronization of individual events in two coupled twin systems. We have shown experimentally and numerically that close to its solitary lasing threshold the power dropouts in the output characteristics of a semiconductor laser with feedback are mostly dominated by deterministic chaos. Our experiments show a fraction $\zeta$ of more than $80 \%$ of the dropouts to be driven deterministically. With increasing pump current $\zeta$ decreases.

Nevertheless, certain limitations of the presented method apply that lead to more unsynchronized dropouts than those which are actually induced by noise and therefore to a smaller synchronized event fraction. We have identified bubbling-induced and mismatch-induced divergences from the single laser dynamics manifold, detection errors and manual classification errors as potential diminishing factors of the deterministic dropout fraction $\zeta$. On an individual event basis we can classify dropouts as deterministically induced with high certainty; the nonsynchronous dropouts can, however, not be characterized further. The experimental results for $\zeta$ have, thus, to be considered a lower limit.

Our numerical results show that the effect of noise becomes more significant for operation close to the lasing threshold if the spontaneous emission factor is increased by one or two orders of magnitude relative to an assumed realistic value of $\beta=10^{-6}$ to $10^{-5}$ for QW single-mode edge-emitting lasers. A higher noise level has a bigger impact on the dynamics close to threshold than for larger pump currents-a smaller fraction of dropouts occurs synchronized close to threshold than for higher currents. It should be noted that the spontaneous emission factor can be of different orders of magnitude for different kinds of lasers (e.g., photonic crystal lasers). Therefore, the fact that most dropouts occur synchronized while operating close to threshold could be modified for different lasers. Our modeling results indicate that for spontaneous emission factors $\beta$ up to the assumed value for the lasers used in the experiments, noise has only little effect on the synchronized dropout fraction when operating the lasers close to threshold. As an example, the value of $\zeta$ varies from $\zeta \approx 1.0$ for $\beta=10^{-9}$ and pump current $I / I_{\mathrm{thr}}=1.002$ to $\zeta \approx 0.93$ for $\beta=10^{-6}$ and the same pump current value. Still, there is a significant decrease in $\zeta$ for larger pump currents due to spontaneous emission. $\zeta$ varies from $\zeta \approx 0.95$ for $\beta \approx 10^{-9}$ and pump current $I / I_{\mathrm{thr}}=1.15$ to $\zeta \approx 0.76$ for $\beta \approx 10^{-6}$.

If one assumes the existence of common dynamical features as precursors of deterministically induced power dropouts, future work could entail classification of events with statistical or pattern-recognition approaches using machine learning concepts [30,31]. The dynamics preceding known deterministic dropouts would be used to train the data processing reservoir. Yet unclassified dropout events would then be fed into the trained reservoir and be classified binarily-deterministically driven or not.

The presented approach can, in general, be applied to other noisy chaotic systems that are synchronizable and exhibit irregular characteristic events (e.g., spikes, dips, and patterns). In a system of coupled neuron populations of the HodgkinHuxley type (see, e.g., [32]) the individual spiking behavior could be analyzed with respect to a deterministic origin using the scheme presented in this paper. As a necessary condition, the synchronized dynamics of two coupled twin subsystems have to be identical to the dynamics of a corresponding single system. If the required symmetry conditions apply, the presented method can even be applied to systems whose governing equations are unknown.

Since bubbling is not present in all coupling schemes of noisy chaotic oscillators, the corresponding fraction $\zeta$ of synchronized characteristic events could even have a higher significance for those kinds of systems.

Our conclusions are strongest the larger the synchronized dropout fraction $\zeta$ is. For the regime with a smaller $\zeta$, one can complement the presented method with statistical measures based on an information theoretical analysis, which are based on different approaches and therefore exhibit other opportunities and limitations. [12]. Combining an individual event-based approach with others based on statistical methods could give a broader access to characterizing semiconductor laser dynamics over a wide range of parameters. This might enable further tailoring of dynamical features and their utilization in applications.

\section{ACKNOWLEDGMENTS}

The authors would like to thank Thomas Jüngling for fruitful discussions. This work was supported by MICINN (Spain) 
under Project TEC2009-14101 (DeCoDicA), by MINECO (Spain) under Project TEC2012-36335 (TRIPHOP), by the Government of the Balearic Islands within Grups Competitius, and by the European Commission under EC FP7 Project
PHOCUS (Grant No. 240763). K.H. acknowledges financial support from the Government of the Balearic Islands (Department of Education, Culture, and Universities), cofunded by the European Social Fund.
[1] M. C. Soriano, J. García-Ojalvo, C. R. Mirasso, and I. Fischer, Rev. Mod. Phys. 85, 421 (2013).

[2] T. Sano, Phys. Rev. A 50, 2719 (1994).

[3] I. Fischer, G. H. M. van Tartwijk, A. M. Levine, W. Elsäßer, E. O. Göbel, and D. Lenstra, Phys. Rev. Lett. 76, 220 (1996).

[4] C. H. Henry and R. F. Kazarinov, IEEE J. Quantum Electron. 22, 294 (1986).

[5] J. Mørk, B. Tromborg, and P. L. Christiansen, IEEE J. Quantum Electron. 24, 123 (1988).

[6] A. Hohl, H. J. C. van der Linden, and R. Roy, Opt. Lett. 20, 2396 (1995).

[7] T. Heil, I. Fischer, and W. Elsäßer, Phys. Rev. A 58, 2672 (1998).

[8] T. Heil, I. Fischer, W. Elässer, J. Mulet, and C. R. Mirasso, Opt. Lett. 24, 1275 (1999).

[9] T. Heil, I. Fischer, and W. Elsäßer, J. Opt. B: Quantum Semiclass. Opt. 2, 413 (2000).

[10] Y. Hong and K. A. Shore, Opt. Lett. 30, 3332 (2005).

[11] J. Tiana-Alsina, J. M. Buldú, M. C. Torrent, and J. García-Ojalvo, Phil. Trans. R. Soc. A 368, 367 (2010).

[12] A. Aragoneses, N. Rubido, J. Tiana-Alsina, M. C. Torrent, and C. Masoller, Sci. Rep. 3, 1778 (2013).

[13] K. Hicke, O. D’Huys, V. Flunkert, E. Schöll, J. Danckaert, and I. Fischer, Phys. Rev. E 83, 056211 (2011).

[14] S. Heiligenthal, T. Jüngling, O. D’Huys, D. A. Arroyo-Almanza, M. C. Soriano, I. Fischer, I. Kanter, and W. Kinzel, Phys. Rev. E 88, 012902 (2013).

[15] R. Vicente, C. R. Mirasso, and I. Fischer, Opt. Lett. 32, 403 (2007).

[16] E. Klein, N. Gross, M. Rosenbluh, W. Kinzel, L. Khaykovich, and I. Kanter, Phys. Rev. E 73, 066214 (2006).
[17] M. Peil, L. Larger, and I. Fischer, Phys. Rev. E 76, 045201(R) (2007).

[18] N. Jiang, W. Pan, B. Luo, L. Yan, S. Xiang, L. Yang, D. Zheng, and N. Li, Phys. Rev. E 81, 066217 (2010).

[19] J. Tiana-Alsina, K. Hicke, X. Porte, M. C. Soriano, M. C. Torrent, J. Garcia-Ojalvo, and I. Fischer, Phys. Rev. E 85, 026209 (2012).

[20] P. Ashwin, J. Buescu, and I. Stewart, Phys. Lett. A 193, 126 (1994).

[21] S. C. Venkataramani, B. R. Hunt, E. Ott, D. J. Gauthier, and J. C. Bienfang, Phys. Rev. Lett. 77, 5361 (1996).

[22] M. Sauer and F. Kaiser, Phys. Lett. A 243, 38 (1998).

[23] N. Platt, E. A. Spiegel, and C. Tresser, Phys. Rev. Lett. 70, 279 (1993).

[24] J. F. Heagy, T. L. Carroll, and L. M. Pecora, Phys. Rev. Lett. 73, 3528 (1994).

[25] E. Ott and J. C. Sommerer, Phys. Lett. A 188, 39 (1994).

[26] V. Flunkert, O. D’Huys, J. Danckaert, I. Fischer, and E. Schöll, Phys. Rev. E 79, 065201(R) (2009).

[27] R. Lang and K. Kobayashi, IEEE J. Quantum Electron. 16, 347 (1980).

[28] G. N. Milshtein, Theor. Prob. Appl. 19(3), 557 (1974).

[29] M. San Miguel and R. Toral, in Instabilities and Nonequilibrium Structures VI, Nonlinear Phenomena and Complex Systems Vol. 5, edited by E. Tirapegui, J. Martinez, and R. Tiemann (Kluwer Academic Publishers, Dordrecht, The Netherlands, 2000), pp. 35-127.

[30] H. Jaeger and H. Haas, Science 304, 78 (2004).

[31] L. Appeltant, M. C. Soriano, G. Van der Sande, J. Danckaert, S. Massar, J. Dambre, B. Schrauwen, C. R. Mirasso, and I. Fischer, Nat. Comm. 2, 468 (2011).

[32] R. Vicente, L. L. Gollo, C. R. Mirasso, I. Fischer, and P. Gordon, Proc. Natl. Acad. Sci. USA 105, 17157 (2008). 